\title{
THE STRAIN-ENERGY FUNCTION FOR ANISOTROPIC ELASTIC MATERIALS
}

BY

\author{
G. F. SMITH AND R. S. RIVLIN( $\left.{ }^{1}\right)$
}

1. Introduction. If we consider a body of perfectly elastic material to undergo deformation in which a point initially at $X_{i}^{\prime}$ in the rectangular Cartesian coordinate system $x_{i}^{\prime}$ moves to $x_{i}^{\prime}$ in the same coordinate system, then the strain-energy function $W$ is a single-valued function of the quantities $g_{i j}^{\prime}$ defined by

$$
g_{i j}^{\prime}=\frac{\partial x_{k}^{\prime}}{\partial X_{i}^{\prime}} \frac{\partial x_{k}^{\prime}}{\partial X_{j}^{\prime}}-\delta_{i j} .
$$

We assume (see Appendix) that $W$ is expressible as a polynomial in the quantities $g_{i j}^{\prime}$ and denote this by

$$
W=W\left(g_{i j}^{\prime}\right) .
$$

We define the displacement components $u_{i}^{\prime}$ by

$$
u_{i}^{\prime}=x_{i}^{\prime}-X_{i}^{\prime} .
$$

Then, if the displacement gradients $\partial u_{i}^{\prime} / \partial X_{j}^{\prime}$ are sufficiently small compared with unity, the strain-energy function $W$, considered as a polynomial in $\partial u_{i}^{\prime} / \partial X_{j}^{\prime}$, may be approximated by the leading terms of first and second degree in $\partial u_{i}^{\prime} / \partial X_{j}^{\prime}$. If we assume that the stress in the material is zero when $u_{i}^{\prime}=0$, then the coefficients of the first degree terms are zero and $W$ is a homogeneous quadratic expression of the form

$$
W=c_{i j l m}\left(\frac{\partial u_{i}^{\prime}}{\partial X_{j}^{\prime}}+\frac{\partial u_{j}^{\prime}}{\partial X_{i}^{\prime}}\right)\left(\frac{\partial u_{l}^{\prime}}{\partial X_{m}^{\prime}}+\frac{\partial u_{m}^{\prime}}{\partial X_{l}^{\prime}}\right),
$$

where $c_{i j l m}$ are constants.

If the material possesses symmetry, certain restrictions, depending on the type of symmetry, are imposed on these constants. These restrictions were fully investigated by Voigt (1910) for the various classes of crystal symmetry. A higher order approximation to $W$, in the case when $\partial u_{i}^{\prime} / \partial X_{j}^{\prime} \ll 1$, is

Received by the editors October 27, 1956.

(1) The results presented in this paper were obtained in the course of research sponsored by the Office of Ordnance Research, U. S. Army, under Contract No. DA-19-020-3487 with Brown University. 
obtained by retaining in the expression for $W$ as a polynomial in $\partial u_{i}^{\prime} / \partial X_{j}^{\prime}$ all terms of third and lower degree in $\partial u_{i}^{\prime} / \partial X_{j}^{\prime}$. Alternatively, in the expression for $W$ as a polynomial in $g_{i j}^{\prime}$ all terms of third or lower degree in $g_{i j}^{\prime}$ may be retained. In both cases, the coefficients of the first degree terms vanish if the stress is assumed zero when $u_{i}^{\prime}=0$. Considering $W$ to be a polynomial of third degree in $g_{i j}^{\prime}$, Birch (1947) has investigated the restrictions imposed on the coefficients in this polynomial if the material considered has cubic symmetry of the hexoctahedral, gyroidal, hextetrahedral, diploidal or tetartoidal classes. Murnaghan (1951) has considered the restrictions imposed on these coefficients by certain basic types of symmetry, e.g. rotational symmetry about an axis. Sheng (1955) has investigated these restrictions for the various crystal classes $\left({ }^{2}\right)$.

In the present paper, we discuss the restrictions imposed by symmetry on the form of the strain-energy function, for elastic materials belonging to the various crystal classes, without in any way restricting the degree of the expression for the strain-energy function as a polynomial in $g_{i j}^{\prime}$. The results are obtained by consistent use of theorems in classical invariant theory, although in certain of the simple cases, e.g. materials having rhombic symmetry, the conclusions can be readily reached by inspection (Green and Wilkes (1954)). It is seen that the 32 crystal classes have strain-energy functions of eleven different types, which coalesce into nine types if the approximations of classical elasticity theory are made.

Throughout this paper, the nomenclature for the various crystal classes is that employed by Dana and Hurlbut (1952).

For each of the crystal classes, a preferred rectangular Cartesian coordinate system $x_{i}$ is chosen and the symmetry of the material is described by the group of transformations which transforms this coordinate system into its equivalent positions. The strain-energy function is assumed to be a polynomial in the six quantities $g_{i j}$, which are the quantities $g_{j}^{i \prime}$ given in (1.1) but defined in the reference system $x_{i}$. This polynomial must, of course, be form-invariant under the transformations describing the particular crystal class considered. Hence, the problem of determining the limitations imposed on the strain-energy function reduces to the determination of a polynomial basis for polynomials in $g_{i j}$ which are form-invariant under the group of transformations associated with the crystal class considered.

Such a polynomial basis $I_{1}, I_{2}, \cdots, I_{n}$ (say) is determined for each of the crystal classes. It has the following properties:

(i) each of the quantities $I_{1}, I_{2}, \cdots, I_{n}$ is a polynomial in $g_{i j}$ which is form-invariant under the group of transformations associated with the crystal class considered and hence any polynomial in $I_{1}, I_{2}, \cdots, I_{n}$ is form invariant under this group of transformations;

(2) Sheng (1955) considers the restrictions imposed on the expressions for the stress by the symmetry of the material. The restrictions on the strain-energy function follow readily from these results. 
(ii) any polynomial in $g_{i j}$ which is form-invariant under the group of transformations, and hence the strain-energy function $W$, is expressible as a polynomial in $I_{1}, I_{2}, \cdots, I_{n}$.

From this knowledge of $W$, we can determine the stress components $\tau_{i j}$ in the coordinate system $x_{i}$ by employing the formula (see, for example, Truesdell (1952) or Green and Zerna (1954))

$$
\tau_{i j}=\frac{1}{\operatorname{det} \frac{\partial x_{p}}{\partial X_{q}}}\left[\frac{\partial x_{i}}{\partial X_{k}} \frac{\partial W}{\partial\left(\partial x_{j} / \partial X_{k}\right)}\right] .
$$

In this formula $x_{i}$ and $X_{i}$ are the positions in the coordinate system $x_{i}$ of a particle of the material in the deformed and undeformed states respectively.

From Equation (1.5) and (ii) it follows immediately that

$$
\tau_{i j}=\frac{1}{\operatorname{det} \frac{\partial x_{p}}{\partial X_{q}}} \sum_{r=1}^{n} \frac{\partial W}{\partial I_{r}} \frac{\partial x_{i}}{\partial X_{k}} \frac{\partial I_{r}}{\partial\left(\partial x_{j} / \partial X_{k}\right)} .
$$

2. Symmetry transformations. For each of the materials considered in this paper, there exist in the undeformed state three preferred directions in the material which may be defined by the unit vectors $h_{i}(i=1,2,3)$. Except for the pedial class of the triclinic system, there are a number of equivalent ways in which the triad of vectors $\boldsymbol{h}_{i}$ may be chosen. The symmetry properties of the material may be defined by the group of orthogonal transformations which transform any of these triads into its equivalent positions.

The following transformations are sufficient for the description of the symmetry properties in the various crystal systems:

$$
\begin{aligned}
& I=(1,1,1), \quad C=(-1,-1,-1), \\
& \boldsymbol{R}_{1}=(-1,1,1), \quad \boldsymbol{R}_{\mathbf{2}}=(1,-1,1), \quad \boldsymbol{R}_{\mathbf{3}}=(1,1,-1) \text {, } \\
& D_{1}=(1,-1,-1), \quad D_{2}=(-1,1,-1), \quad D_{3}=(-1,-1,1), \\
& T_{1}=\left\|\begin{array}{lll}
1, & 0, & 0 \\
0, & 0, & 1 \\
0, & 1, & 0
\end{array}\right\|, \quad T_{2}=\left\|\begin{array}{ccc}
0, & 0, & 1 \\
0, & 1, & 0 \\
1, & 0, & 0
\end{array}\right\|, \quad T_{3}=\left\|\begin{array}{ccc}
0, & 1, & 0 \\
1, & 0, & 0 \\
0, & 0, & 1
\end{array}\right\| \text {, } \\
& M_{1}=\left\|\begin{array}{lll}
0, & 1, & 0 \\
0, & 0, & 1 \\
1, & 0, & 0
\end{array}\right\|, \quad M_{2}=\left\|\begin{array}{lll}
0, & 0, & 1 \\
1, & 0, & 0 \\
0, & 1, & 0
\end{array}\right\| \text {, } \\
& S_{1}=\left\|\begin{array}{ccc}
-1 / 2, & 3^{1 / 2} / 2, & 0 \\
-3^{1 / 2} / 2, & -1 / 2, & 0 \\
0, & 0, & 1
\end{array}\right\| \text { and } S_{2}=\left\|\begin{array}{ccc}
-1 / 2, & -3^{1 / 2} / 2, & 0 \\
3^{1 / 2} / 2, & -1 / 2, & 0 \\
0, & 0, & 1
\end{array}\right\| \text {. }
\end{aligned}
$$


In Equation (2.1), the notation

$$
(a, b, c)=\left\|\begin{array}{lll}
a, & 0, & 0 \\
0, & b, & 0 \\
0, & 0, & c
\end{array}\right\|
$$

is used. $I$ is the identity transformation. $C$ is the central inversion transformation. $\boldsymbol{R}_{j}$ is the reflection transformation which transforms a rectangular Cartesian coordinate system $x_{i}$, the $x_{j}$ axis of which lies in the direction of the unit vector $\boldsymbol{h}_{j}$, into its image in the plane normal to $\boldsymbol{h}_{j}$. The transformation $D_{j}$ transforms this coordinate system into that obtained by rotating it through $180^{\circ}$ about the $x_{j}$ axis. The transformation $T_{j}$ transforms a rectangular Cartesian coordinate system $x_{i}$, the axes of which lie in the directions of the unit vectors $h_{i}$, into the coordinate system obtained by reflecting the system $x_{i}$ in a plane passing through the axis $x_{j}$ and bisecting the angle between the remaining two axes. The transformations $\boldsymbol{M}_{1}$ and $\boldsymbol{M}_{2}$ transform a rectangular Cartesian coordinate system $x_{i}$, the axes of which lie in the directions of $h_{i}$, into the systems obtained by rotating the system $x_{i}$ through $120^{\circ}$ and $240^{\circ}$ respectively about a line passing through the origin and the point $(1,1,1)$. The transformations $\boldsymbol{S}_{1}$ and $\boldsymbol{S}_{2}$ transform a rectangular Cartesian coordinate system $x_{i}$, the $x_{3}$ axis of which lies in the direction of $h_{3}$ into the systems obtained by rotation of this coordinate system through $120^{\circ}$ and $240^{\circ}$ respectively about the $x_{3}$ axis.

3. Form-invariance of the strain-energy function. For each of the symmetry classes that we consider, we shall choose as reference system a rectangular Cartesian coordinate system $x_{i}$, so related to the preferred directions $h_{i}$ in the material under consideration that the symmetry of the material may be described by one or more of the transformations (2.1). Let $X_{i}$ and $x_{i}$ denote the positions of a particle of the material in this coordinate system in the undeformed and deformed states respectively. Then, from (1.1), it is seen that the strain-energy function $W$ is expressible as a polynomial in the quantities $g_{i j}$ defined by

$$
g_{i j}=\frac{\partial x_{k}}{\partial X_{i}} \frac{\partial x_{k}}{\partial X_{j}}-\delta_{i j}
$$

We shall express this as

$$
W=W\left(g_{i j}\right) .
$$

Let $\bar{x}_{\imath}$ be the coordinate system into which the system $x_{i}$ is transformed by a transformation $\left\|t_{i j}\right\|$ of the group describing the symmetry properties of the material under consideration. Then, if $\bar{X}_{i}$ and $\bar{x}_{i}$ are the coordinates in the system $\bar{x}_{i}$ corresponding to the coordinates $X_{i}$ and $x_{i}$ in the system $x_{i}$, we have 


$$
\bar{X}_{i}=t_{i j} X_{j} \quad \text { and } \quad \bar{x}_{i}=t_{i j} x_{j} .
$$

Defining $\bar{g}_{i j}$ by

$$
\bar{g}_{i j}=\frac{\partial \bar{x}_{k}}{\partial \bar{X}_{i}} \frac{\partial \bar{x}_{k}}{\partial \bar{X}_{j}}-\delta_{i j},
$$

we have

$$
\bar{g}_{i j}=t_{i p} t_{j q} g_{p q} .
$$

Now, since the coordinate systems $x_{i}$ and $\bar{x}_{i}$ are equivalent, we must have

$$
W\left(g_{i j}\right)=W\left(\bar{g}_{i j}\right),
$$

i.e. the strain-energy function must be form-invariant under any transformation of the group defining the symmetry properties of the material. It is the object of this paper to determine the limitations imposed on $W$ by the condition (3.6), with (3.5), for each of the classes of the crystal systems. For each crystal class, the condition (3.6) must, of course, be satisfied for every trans-

\begin{tabular}{|c|c|c|c|c|c|c|c|}
\hline Line No. & $\left\|t_{i j}\right\|$ & $\bar{g}_{11}$ & $\bar{g}_{22}$ & $\bar{g}_{33}$ & $\bar{g}_{23}$ & $\bar{g}_{31}$ & $\bar{g}_{12}$ \\
\hline 1 & $I, C$ & $g_{11}$ & $g_{22}$ & $g_{33}$ & $g_{23}$ & $g_{31}$ & $g_{12}$ \\
\hline 2 & $R_{1}, D_{1}$ & $g_{11}$ & $g_{22}$ & $g_{33}$ & $g_{23}$ & $-g_{31}$ & $-g_{12}$ \\
\hline 3 & $R_{2}, D_{2}$ & $g_{11}$ & $g_{22}$ & $g_{33}$ & $-g_{23}$ & $g_{31}$ & $-g_{12}$ \\
\hline 4 & $R_{3}, D_{3}$ & $g_{11}$ & $g_{22}$ & $g_{33}$ & $-g_{23}$ & $-g_{31}$ & $g_{12}$ \\
\hline 5 & $T_{1}, C T_{1}$ & $g_{11}$ & $g_{33}$ & $g_{22}$ & $g_{23}$ & $g_{12}$ & $g_{31}$ \\
\hline 6 & $R_{1} T_{1}, D_{1} T_{1}$ & $g_{11}$ & $g_{33}$ & $g_{22}$ & $g_{23}$ & $-g_{12}$ & $-g_{31}$ \\
\hline 7 & $R_{2} T_{1}, D_{2} T_{1}$ & $g_{11}$ & $g_{33}$ & $g_{22}$ & $-g_{23}$ & $g_{12}$ & $-g_{31}$ \\
\hline 8 & $R_{3} T_{1}, D_{3} T_{1}$ & $g_{11}$ & $g_{33}$ & $g_{22}$ & $-g_{23}$ & $-g_{12}$ & $g_{31}$ \\
\hline 9 & $T_{2}, C T_{2}$ & $g_{33}$ & $g_{22}$ & $g_{11}$ & $g_{12}$ & $g_{31}$ & $g_{23}$ \\
\hline 10 & $R_{1} T_{2}, D_{1} T_{2}$ & $g_{33}$ & $g_{22}$ & $g_{11}$ & $g_{12}$ & $-g_{31}$ & $-g_{23}$ \\
\hline 11 & $R_{2} T_{2}, D_{2} T_{2}$ & $g_{33}$ & $g_{22}$ & $g_{11}$ & $-g_{12}$ & $g_{31}$ & $-g_{23}$ \\
\hline 12 & $R_{3} T_{2}, D_{3} T_{2}$ & $g_{33}$ & $g_{22}$ & $g_{11}$ & $-g_{12}$ & $-g_{31}$ & $g_{23}$ \\
\hline 13 & $T_{3}, C T_{3}$ & $g_{22}$ & $g_{11}$ & $g_{33}$ & $g_{31}$ & $g_{23}$ & $g_{12}$ \\
\hline 14 & $R_{1} T_{3}, D_{1} T_{3}$ & $g_{22}$ & $g_{11}$ & $g_{33}$ & $g_{31}$ & $-g_{23}$ & $-g_{12}$ \\
\hline 15 & $R_{2} T_{3}, D_{2} T_{3}$ & $g_{22}$ & $g_{11}$ & $g_{33}$ & $-g_{31}$ & $g_{23}$ & $-g_{12}$ \\
\hline 16 & $R_{3} T_{3}, D_{3} T_{3}$ & $g_{22}$ & $g_{11}$ & $g_{33}$ & $-g_{31}$ & $-g_{23}$ & $g_{12}$ \\
\hline 17 & $M_{1}, C M_{1}$ & $g_{22}$ & $g_{33}$ & $g_{11}$ & $g_{31}$ & $g_{12}$ & $g_{23}$ \\
\hline 18 & $R_{1} M_{1}, D_{1} M_{1}$ & $g_{22}$ & $g_{33}$ & $g_{11}$ & $g_{31}$ & $-g_{12}$ & $-g_{23}$ \\
\hline 19 & $R_{2} M_{1}, D_{2} M_{1}$ & $g_{22}$ & $g_{33}$ & $g_{11}$ & $-g_{31}$ & $g_{12}$ & $-g_{23}$ \\
\hline 20 & $R_{3} M_{1}, D_{3} M_{1}$ & $g_{22}$ & $g_{33}$ & $g_{11}$ & $-g_{31}$ & $-g_{12}$ & $g_{23}$ \\
\hline 21 & $M_{2}, C M_{2}$ & $g_{33}$ & $g_{11}$ & $g_{\Sigma 2}$ & $g_{12}$ & $g_{23}$ & $g_{31}$ \\
\hline 22 & $R_{1} M_{2}, D_{1} M_{2}$ & $g_{33}$ & $g_{11}$ & $g_{22}$ & $g_{12}$ & $-g_{23}$ & $-g_{31}$ \\
\hline 23 & $R_{2} M_{2}, D_{2} M_{2}$ & $g_{33}$ & $g_{11}$ & $g_{22}$ & $-g_{12}$ & $g_{23}$ & $-g_{31}$ \\
\hline 24 & $R_{3} M_{2}, D_{3} M_{2}$ & $g_{33}$ & $g_{11}$ & $g_{22}$ & $-g_{12}$ & $-g_{23}$ & $g_{31}$ \\
\hline
\end{tabular}
formation $\left\|t_{i j}\right\|$ of the group describing the symmetry of the material. In Tables 1 and 2, the values of $\bar{g}_{i j}$, obtained from (3.5), are given in terms of

TABLE 1 
$g_{p q}$ for each of the transformations entering into the description of the symmetry properties of the various crystal classes. These transformations are either those given in (2.1) or their products. In Table 2 the notation

$$
y_{1}=g_{11}, \quad y_{2}=\frac{1}{4} g_{11}-\frac{3^{1 / 2}}{2} g_{12}+\frac{3}{4} g_{22}, \quad y_{3}=\frac{1}{4} g_{11}+\frac{3^{1 / 2}}{2} g_{12}+\frac{3}{4} g_{22},
$$

$$
z_{1}=g_{31}, \quad z_{2}=-\frac{1}{2} g_{31}+\frac{3^{1 / 2}}{2} g_{23}, \quad z_{3}=-\frac{1}{2} g_{31}-\frac{3^{1 / 2}}{2} g_{23}
$$

is used. It is, of course, evident that

$$
z_{1}+z_{2}+z_{3}=0 .
$$

The limitation imposed on $W$ by the requirement that it be form-invariant under one of the transformations is obtained from (3.6) by substituting for $\bar{g}_{i j}$ the values appropriate to that transformation given in the tables.

TABLE 2

\begin{tabular}{c|c|c|c|c|c|c|c}
\hline \hline $\begin{array}{c}\text { Line } \\
\text { No. }\end{array}$ & $\left\|t_{i j}\right\|$ & $\bar{g}_{11}$ & $\bar{g}_{22}$ & $\bar{g}_{33}$ & $\bar{g}_{23}$ & $\bar{g}_{31}$ & $\bar{g}_{12}$ \\
\hline 1 & $S_{1}, C S_{1}$ & $y_{2}$ & $\frac{1}{3}\left(2 y_{1}-y_{2}+2 y_{3}\right)$ & $g_{33}$ & $-\frac{1}{3^{1 / 2}}\left(z_{1}-z_{3}\right)$ & $z_{2}$ & $\frac{1}{3^{1 / 2}}\left(y_{1}-y_{3}\right)$ \\
\hline 2 & $S_{2}, C S_{2}$ & $y_{3}$ & $\frac{1}{3}\left(2 y_{1}+2 y_{2}-y_{3}\right)$ & $g_{33}$ & $\frac{1}{3^{1 / 2}}\left(z_{1}-z_{2}\right)$ & $z_{3}$ & $\frac{1}{3^{1 / 2}}\left(y_{2}-y_{1}\right)$ \\
3 & $R_{1} S_{1}, D_{1} S_{1}$ & $y_{2}$ & $\frac{1}{3}\left(2 y_{1}-y_{2}+2 y_{3}\right)$ & $g_{33}$ & $-\frac{1}{3^{1 / 2}}\left(z_{1}-z_{3}\right)$ & $-z_{2}$ & $\frac{1}{3^{1 / 2}}\left(y_{3}-y_{1}\right)$ \\
4 & $R_{1} S_{2}, D_{1} S_{2}$ & $y_{3}$ & $\frac{1}{3}\left(2 y_{1}+2 y_{2}-y_{3}\right)$ & $g_{33}$ & $\frac{1}{3^{1 / 2}}\left(z_{1}-z_{2}\right)$ & $-z_{3}$ & $\frac{1}{3^{1 / 2}}\left(y_{1}-y_{2}\right)$ \\
5 & $R_{2} S_{1}, D_{2} S_{1}$ & $y_{2}$ & $\frac{1}{3}\left(2 y_{1}-y_{2}+2 y_{3}\right)$ & $g_{33}$ & $\frac{1}{3^{1 / 2}}\left(z_{1}-z_{3}\right)$ & $z_{2}$ & $\frac{1}{3^{1 / 2}}\left(y_{3}-y_{1}\right)$ \\
6 & $R_{2} S_{2}, D_{2} S_{2}$ & $y_{3}$ & $\frac{1}{3}\left(2 y_{1}+2 y_{2}-y_{3}\right)$ & $g_{33}$ & $-\frac{1}{3^{1 / 2}}\left(z_{1}-z_{2}\right)$ & $z_{3}$ & $\frac{1}{3^{1 / 2}}\left(y_{1}-y_{2}\right)$ \\
7 & $R_{3} S_{1}, D_{3} S_{1}$ & $y_{2}$ & $\frac{1}{3}\left(2 y_{1}-y_{2}+2 y_{3}\right)$ & $g_{33}$ & $\frac{1}{3^{1 / 2}}\left(z_{1}-z_{3}\right)$ & $-z_{2}$ & $\frac{1}{3^{1 / 2}}\left(y_{1}-y_{3}\right)$ \\
8 & $R_{3} S_{2}, D_{3} S_{2}$ & $y_{3}$ & $\frac{1}{3}\left(2 y_{1}+2 y_{2}-y_{3}\right)$ & $g_{33}$ & $-\frac{1}{3^{1 / 2}}\left(z_{1}-z_{2}\right)$ & $-z_{3}$ & $\frac{1}{3^{1 / 2}}\left(y_{2}-y_{1}\right)$ \\
\hline
\end{tabular}

4. Some theorems on polynomial bases. It has been seen that the requirement that the strain-energy function for a particular crystal class, regarded as a polynomial in the quantities $g_{i j}$ defined by (3.1), be form-invariant under the transformations associated with that class imposes on $W$ a number of restrictions of the type expressed by (3.6) and (3.5). It is well-known that polynomials in a number of variables which are form-invariant under a finite 
group of transformations possess a finite polynomial basis, such that each of the polynomials can be expressed as a polynomial in the elements of the basis and any polynomial in the elements of the basis is form-invariant under the group of transformations. Thus, in order to determine explicitly the limitations imposed on the form of $W$ by the requirement of form-invariance under the group of transformations characterizing a crystal class, we have to determine a polynomial basis for $W$ regarded as a function of the quantities $g_{i j}$. This will be done for each of the crystal classes in $\S \S 5$ to 10 . It will be seen there that for each of the crystal classes the restrictions imposed on $W$ are equivalent to the restriction that it be form-invariant under some subgroup of the full symmetric group of permutations of a number of linear combinations of the six quantities $g_{i j}$. In this section we shall give some theorems regarding the determination of a polynomial basis for polynomials in a number of variables which are form-invariant under certain sub-groups of the full symmetric group of permutations.

Theorem 1. A polynomial basis for polynomials which are symmetric in the two sets of variables $\left(y_{1}, y_{2}, \cdots, y_{n}\right)$ and $\left(z_{1}, z_{2}, \cdots, z_{n}\right)$ is formed by the quantities

$$
\begin{aligned}
I_{j} & =\left(y_{j}+z_{j}\right) / 2 & (j & =1,, 2 \cdots, n), \\
I_{j k} & =\left(y_{j} z_{k}+y_{k} z_{j}\right) / 2 & (j, k & =1,2, \cdots, n) .
\end{aligned}
$$

THEOREM 2. A polynomial basis for polynomials which are symmetric in the three pairs of variables $\left(y_{1}, z_{1}\right),\left(y_{2}, z_{2}\right)$ and $\left(y_{3}, z_{3}\right)$ is formed by the quantities,

$$
\begin{aligned}
& J_{1}=y_{1}+y_{2}+y_{3}, \quad J_{2}=y_{2} y_{3}+y_{3} y_{1}+y_{1} y_{2}, \quad J_{3}=y_{1} y_{2} y_{3}, \\
& J_{4}=z_{1}+z_{2}+z_{3}, \quad J_{5}=z_{2} z_{3}+z_{3} z_{1}+z_{1} z_{2}, \quad J_{6}=z_{1} z_{2} z_{3}, \\
& J_{7}=y_{2} z_{3}+y_{3} z_{2}+y_{3} z_{1}+y_{1} z_{3}+y_{1} z_{2}+y_{2} z_{1}, \\
& J_{8}=y_{1} z_{2} z_{3}+y_{2} z_{3} z_{1}+y_{3} z_{1} z_{2}, \quad J_{9}=z_{1} y_{2} y_{3}+z_{2} y_{3} y_{1}+z_{3} y_{1} y_{2} .
\end{aligned}
$$

Theorems 1 and 2 follow immediately from a well-known theorem in the theory of invariants (see, for example, H. Weyl, 1946, p. 36 et seq.).

Theorem 3. A polynomial basis for polynomials in the variables $y_{1}, y_{2}, y_{3}$, $z_{1}, z_{2}, z_{3}$ which are form-invariant under cyclic rotation of the subscripts $1,2,3$ is formed by the quantities

$$
\begin{aligned}
K_{1} & =y_{1}+y_{2}+y_{3}, \quad K_{2}=y_{2} y_{3}+y_{3} y_{1}+y_{1} y_{2}, \quad K_{3}=y_{1} y_{2} y_{3}, \\
K_{4} & =z_{1}+z_{2}+z_{3}, \quad K_{5}=z_{2} z_{3}+z_{3} z_{1}+z_{1} z_{2}, \quad K_{6}=z_{1} z_{2} z_{3}, \\
K_{7} & =y_{2} z_{3}+y_{3} z_{1}+y_{1} z_{2}, \quad K_{8}=z_{2} y_{3}+z_{3} y_{1}+z_{1} y_{2}, \\
K_{9} & =y_{3} y_{2}^{2}+y_{1} y_{3}^{2}+y_{2} y_{1}^{2}, \quad K_{10}=z_{3} z_{2}^{2}+z_{1} z_{3}^{2}+z_{2} z_{1}^{2} \\
K_{11} & =y_{1} z_{2} z_{3}+y_{2} z_{3} z_{1}+y_{3} z_{1} z_{2}, \quad K_{12}=z_{1} y_{2} y_{3}+z_{2} y_{3} y_{1}+z_{3} y_{1} y_{2}, \\
K_{13} & =y_{1} y_{2} z_{2}+y_{2} y_{3} z_{3}+y_{3} y_{1} z_{1}, \quad K_{14}=z_{1} z_{2} y_{2}+z_{2} z_{3} y_{3}+z_{3} z_{1} y_{1} .
\end{aligned}
$$


This theorem follows almost immediately from the fact that the degree of the elements of a polynomial basis for polynomials which are forminvariant under a finite group of transformations is less than or equal to the number of transformations in that group (see, for example, H. Weyl, 1946, p. 276, et seq.). In this case, there are three transformations in the transformation group, and hence the elements of the polynomial basis are of degree three or lower. With this fact, it can readily be shown that the given set of invariants forms a basis.

Theorem 4. A polynomial basis for polynomials in the variables $y_{1}, y_{2}, \cdots$, $y_{n}, z_{1}, z_{2}, \cdots, z_{n}, I_{1}, I_{2}, \cdots, I_{r}$, which are form-invariant under a group of transformations for which $I_{1}, I_{2}, \cdots, I_{r}$ are invariants, is formed by adjoining to the quantities $I_{1}, I_{2}, \cdots, I_{r}$ a polynomial basis for polynomials in the variables $y_{1}, y_{2}, \cdots, y_{n}, z_{1}, z_{2}, \cdots, z_{n}$ which are form-invariant under the given group of transformations.

The validity of this result is immediately obvious.

5. The triclinic system. For a material having triclinic symmetry, there is no restriction on the orientation of the unit vectors $h_{1}, h_{2}$ and $h_{3}$ defining the preferred directions. We may therefore choose any rectangular Cartesian coordinate system $x_{i}$ as a reference system.

The triclinic system contains two crystal classes. These are the pedial class, the strain-energy function for which must be form-invariant under the identity transformation $I$, and the pinacoidal class, the strain-energy function for which must be form-invariant under the transformation $I$ and the central inversion transformation $\boldsymbol{C}$.

It is seen from Table 1 that neither of the transformations $I$ and $C \mathrm{im}$ poses any restriction on the form of $W$. Hence, the six quantities $g_{i j}$ form a polynomial basis for the triclinic system.

6. The monoclinic system. For a material having monoclinic symmetry, the unit vectors $h_{2}$ and $h_{3}$ are not at right-angles and the unit vector $h_{1}$ is perpendicular to the plane defined by $h_{2}$ and $h_{3}$. We take as our reference system a rectangular Cartesian coordinate system $x_{i}$, the $x_{1}$ axis of which is parallel to $h_{1}$. The axes $x_{2}$ and $x_{3}$ may be in arbitrary perpendicular directions in the $h_{2} h_{3}$ plane.

The monoclinic system contains three classes which, together with the symmetry transformations defining them, are: domatic $\left(I, R_{1}\right)$, sphenoidal $\left(I, D_{1}\right)$, prismatic, $\left(I, C, R_{1}, D_{1}\right)$.

It is seen from Table 1 that, for each of the three crystal classes of the monoclinic system, the limitation imposed on the form of $W$ is given by

$$
W\left(g_{11}, g_{22}, g_{33}, g_{23}, g_{31}, g_{12}\right)=W\left(g_{11}, g_{22}, g_{33}, g_{23},-g_{31},-g_{12}\right) \text {. }
$$

Taking 
and

$$
\begin{aligned}
& \left(y_{1}, y_{2}, \cdots, y_{n}\right)=\left(g_{31}, g_{12}\right), \\
& \left(z_{1}, z_{2}, \cdots, z_{n}\right)=\left(-g_{31},-g_{12}\right)
\end{aligned}
$$

$$
\left(I_{1}, I_{2}, \cdots, I_{r}\right)=\left(g_{11}, g_{22}, g_{33}, g_{23}\right),
$$

in Theorems 1 and 4 , it follows that the quantities

$$
g_{11}, g_{22}, g_{33}, g_{23}, \underset{g_{31}}{2}, g_{12}^{2} \text { and } g_{12} g_{31}
$$

form a polynomial basis for $W$.

7. The rhombic system. For a material having rhombic symmetry, the unit vectors $h_{i}$ are mutually perpendicular. We take as our reference system a rectangular Cartesian coordinate system $x_{i}$, the axes of which are parallel to the vectors $h_{i}$.

The rhombic system contains three classes which, together with the symmetry transformations characterizing them, are: rhombic-pyramidal $\left(I, R_{2}, R_{3}, D_{1}\right)$, rhombic-disphenoidal $\left(I, D_{1}, D_{2}, D_{3}\right)$, rhombic-dipyramidal (I, C, $R_{1}, R_{2}, R_{3}, D_{1}, D_{2}, D_{3}$ ).

It is seen that for each of these classes there are three restrictions on the form of $W$ obtained from (3.6) by giving $\bar{g}_{i j}$ the values in lines 2,3 and 4 respectively of Table 1 . We have already seen in $\S 6$ that the first of these implies that

$$
W=W^{\prime}\left(g_{11}, g_{22}, g_{33}, \underset{g}{g}, \stackrel{2}{g_{31}}, \underset{g_{12}}{2}, g_{12} g_{31}\right),
$$

where $W^{\prime}$ denotes a polynomial in the indicated variables. Now,

$$
W^{\prime}\left(g_{i j}\right)=W^{\prime}\left(\bar{g}_{i j}\right),
$$

where $\bar{g}_{i j}$ is given by the quantities in line 3 of Table 1 . Thus,

$$
\begin{aligned}
& W^{\prime}\left(g_{11}, g_{22}, g_{33}, g_{23}, \underset{g_{31}}{2}, \underset{g_{12}}{2}, g_{12} g_{31}\right) \\
& =W^{\prime}\left(g_{11}, g_{22}, g_{33},-g_{23}, \underset{g_{31}}{2}, \stackrel{2}{g_{12}},-g_{12} g_{31}\right) .
\end{aligned}
$$

Taking, in Theorems 1 and 4,

$$
\begin{aligned}
& \left(y_{1}, y_{2}, \cdots, y_{n}\right)=\left(g_{23}, g_{12} g_{31}\right), \\
& \left(z_{1}, z_{2}, \cdots, z_{n}\right)=\left(-g_{23},-g_{12} g_{31}\right), \\
& \left(I_{1}, I_{2}, \cdots, I_{r}\right)=\left(g_{11}, g_{22}, g_{33}, g_{31}, g_{12}^{2}\right),
\end{aligned}
$$

we see that $W$ must be expressible as a polynomial in

$$
g_{11}, g_{22}, g_{33}, \underset{g_{23}}{2}, g_{31}^{2}, g_{12}^{2} \text { and } g_{23} g_{31} g_{12} \text {. }
$$

It is readily seen that any such polynomial satisfies the restriction obtained 
from (3.6) by giving $\bar{g}_{i j}$ the values in line 4 of Table 1 . Hence, the quantities (7.5) form a polynomial basis for $W$.

8. The tetragonal system. For a material having tetragonal symmetry, the unit vectors $h_{i}$ are mutually perpendicular. As in the case of the rhombic system, we take as our reference system a rectangular Cartesian coordinate system $x_{i}$, the axes of which are parallel to the vectors $h_{i}$. We shall take the $x_{3}$ axis to be the principal axis of symmetry.

The tetragonal system contains seven crystal classes which, together with the transformation groups characterizing them, are:
tetragonal-disphenoidal
tetragonal-pyramidal
$\left(I, D_{3}, D_{1} T_{3}, D_{2} T_{3}\right)$,
tetragonal-dipyramidal
$\left(I, D_{3}, R_{1} T_{3}, R_{2} T_{3}\right)$,
tetragonal-scalenohedral
ditetragonal-pyramidal
tetragonal-trapezohedral
(I, C, $R_{3}, D_{3}, R_{1} T_{3}, R_{2} T_{3}, D_{1} T_{3}, D_{2} T_{3}$ ),
(I, $\left.D_{1}, D_{2}, D_{3}, T_{3}, D_{1} T_{3}, D_{2} T_{3}, D_{3} T_{3}\right)$,
$\left(I, R_{1}, R_{2}, D_{3}, T_{3}, R_{1} T_{3}, R_{2} T_{3}, D_{3} T_{3}\right)$,
ditetragonal-dipyramidal
( $I, D_{1}, D_{2}, D_{3}, C T_{3}, R_{1} T_{3}, R_{2} T_{3}, R_{3} T_{3}$ ),
$\left(I, C, R_{1}, R_{2}, R_{3}, D_{1}, D_{2}, D_{3}, T_{3}, C T_{3}, R_{1} T_{3}, R_{2} T_{3}\right.$, $R_{3} T_{3}, D_{1} T_{3}, D_{2} T_{3}, D_{3} T_{3}$ ).

It is seen that for each of the first three classes of the tetragonal system listed above (tetragonal-disphenoidal, tetragonal-pyramidal, tetragonaldipyramidal), the limitations on $W$ are those obtained by substituting for $\bar{g}_{i j}$ in (3.6) from Lines 4,14 and 15 respectively of Table 1 . It can be shown in a manner analogous to that adopted in discussing the monoclinic system that the first of these limitations implies that

$$
W\left(g_{i j}\right)=W^{\prime}\left(g_{11}, g_{22}, g_{33}, \stackrel{2}{g_{23}}, \stackrel{2}{g_{31}}, g_{12}, g_{23} g_{31}\right),
$$

where $W^{\prime}$ is a polynomial in the indicated variables. With (8.1), the restriction derived from line 14 of Table 1 yields

$$
\begin{aligned}
& W^{\prime}\left(g_{11}, g_{22}, g_{33}, \underset{g_{23}}{2}, \stackrel{2}{g_{31}}, g_{12}, g_{23} g_{31}\right) \\
& \quad=W^{\prime}\left(g_{22}, g_{11}, g_{33}, \underset{g_{31}}{2}, \underset{g_{23}}{2},-g_{12},-g_{23} g_{31}\right) .
\end{aligned}
$$

Employing Theorems 1 and 4 as before and omitting redundant elements, we see that $W^{\prime}$ and hence $W$ is expressible as a polynomial in

$$
\begin{aligned}
& g_{11}+g_{22}, g_{33}, \stackrel{2}{g} g_{23}+g_{31}^{2}, \stackrel{2}{g_{12}}, g_{11} g_{22}, g_{12}\left(g_{11}-g_{22}\right), \\
& g_{23} g_{31}\left(g_{11}-g_{22}\right), g_{23} g_{31} g_{12}, g_{12}\left(g_{31}^{2}-g_{23}^{2}\right), \\
& g_{11} g_{23}^{2}+g_{22} g_{31}^{2}, g_{23} g_{31}\left(g_{31}^{2}-g_{23}^{2}\right) \text { and } \underset{g_{23} g_{31}^{2}}{2} \text {. }
\end{aligned}
$$

Since such a polynomial satisfies the limitation derived from line 15 of Table 1 , we see that the quantities (8.3) form a polynomial basis for the first three classes of the tetragonal system. 
For the remaining four classes of the tetragonal system, the restrictions imposed on $W$ are obtained by substituting in (3.6) from lines $2,3,4,13$, 14,15 and 16 respectively of Table 1 . In the case of the rhombic system it was seen that the first three of these restrictions implies that $W$ is a polynomial $W^{\prime}$ (say) in the quantities (7.5). Introducing the limitation derived from line 13 of Table 1 and employing Theorems 1 and 4 as before, we see that $W^{\prime}$ and hence $W$ must be expressible as a polynomial in

$$
g_{11}+g_{22}, \underset{g}{g}, \stackrel{2}{g_{23}}+\stackrel{2}{g_{31}}, \stackrel{2}{g_{12}}, g_{11} g_{22}, g_{23} g_{31} g_{12}, \underset{g_{11} g_{23}^{2}}{2}+\underset{g_{22} g_{31}}{2} \text { and } \underset{g_{23} g_{31}}{2} \text {. }
$$

Such a polynomial automatically satisfies the limitations derived from lines 14,15 and 16 of Table 1 and hence forms a polynomial basis for the tetragonalscalenohedral, ditetragonal-pyramidal, tetragonal-trapezohedral and ditetragonal-dipyramidal classes.

9. The cubic system. For a material having cubic symmetry the unit vectors $h_{1}, h_{2}$ and $h_{3}$ are mutually perpendicular. We take as our reference system a rectangular Cartesian coordinate system $x_{i}$, the axes of which are parallel to the vectors $h_{i}$.

The cubic system contains five classes which, together with the symmetry transformations characterizing them, are:

tetartoidal $\quad\left(I, D_{1}, D_{2}, D_{3}, M_{1}, D_{1} M_{1}, D_{2} M_{1}, D_{3} M_{1}, M_{2}, D_{1} M_{2}, D_{2} M_{2}\right.$, $\left.D_{3} M_{2}\right)$,

diploidal $\quad\left(I, C, R_{1}, R_{2}, R_{3}, D_{1}, D_{2}, D_{3}, M_{1}, C M_{1}, R_{1} M_{1}, R_{2} M_{1}, R_{3} M_{1}\right.$, $D_{1} M_{1}, D_{2} M_{1}, D_{3} M_{1}, M_{2}, C M_{2}, R_{1} M_{2}, R_{2} M_{2}, R_{3} M_{2}, D_{1} M_{2}$, $\left.D_{2} M_{2}, D_{3} M_{2}\right)$,

hextetrahedral ( $I, D_{1}, D_{2}, D_{3}, T_{1}, D_{1} T_{1}, D_{2} T_{1}, D_{3} T_{1}, T_{2}, D_{1} T_{2}, D_{2} T_{2}, D_{3} T_{2}, T_{3}$, $D_{1} T_{3}, D_{2} T_{3}, D_{3} T_{3}, M_{1}, D_{1} M_{1}, D_{2} M_{1}, D_{3} M_{1}, M_{2}, D_{1} M_{2}, D_{2} M_{2}$, $\left.D_{3} M_{2}\right)$,

gyroidal

(I, $D_{1}, D_{2}, D_{3}, C T_{1}, R_{1} T_{1}, R_{2} T_{1}, R_{3} T_{1}, C T_{2}, R_{1} T_{2}, R_{2} T_{2}, R_{3} T_{2}$, $C T_{3}, R_{1} T_{3}, R_{2} T_{3}, R_{3} T_{3}, M_{1}, D_{1} M_{1}, D_{2} M_{1}, D_{3} M_{1}, M_{2}, D_{1} M_{2}$, $\left.D_{2} M_{2}, D_{3} M_{2}\right)$,

hexoctahedral ( $I, C, R_{1}, R_{2}, R_{3}, D_{1}, D_{2}, D_{3}, T_{1}, C T_{1}, R_{1} T_{1}, R_{2} T_{1}, R_{3} T_{1}, D_{1} T_{1}$, $D_{2} T_{1}, D_{3} T_{1}, T_{2}, C T_{2}, R_{1} T_{2}, R_{2} T_{2}, R_{3} T_{2}, D_{1} T_{2}, D_{2} T_{2}, D_{3} T_{2}, T_{3}$, $C T_{3}, R_{1} T_{3}, R_{2} T_{3}, R_{3} T_{3}, D_{1} T_{3}, D_{2} T_{3}, D_{3} T_{3}, M_{1}, C M_{1}, R_{1} M_{1}$, $R_{2} M_{1}, R_{3} M_{1}, D_{1} M_{1}, D_{2} M_{1}, D_{3} M_{1}, M_{2}, C M_{2}, R_{1} M_{2}, R_{2} M_{2}$, $\left.R_{3} M_{2}, D_{1} M_{2}, D_{2} M_{2}, D_{3} M_{2}\right)$.

(i) Tetartoidal and diploidal classes.

It is seen that for each of these crystal classes the limitations imposed on $W$ are obtained by substituting in (3.6) for $\bar{g}_{i j}$ from lines 2, 3, 4 and lines 17 to 24 respectively of Table 1 . As in the case of the rhombic system, it is seen that the restrictions obtained from lines 2,3 and 4 imply that $W$ is a polynomial $W^{\prime}$ (say) in the quantities (7.5). It is readily seen that the restrictions obtained from lines 17 to 24 imply that $W^{\prime}$ must satisfy the relations 


$$
\begin{aligned}
& W^{\prime}\left(g_{11}, g_{22}, g_{33}, \stackrel{2}{g_{23}}, \stackrel{2}{g_{31}}, \stackrel{2}{g_{12}}, g_{23} g_{31} g_{12}\right) \\
& \quad=W^{\prime}\left(g_{22}, g_{33}, g_{11}, \stackrel{2}{g_{31}} \underset{g_{12}}{2}, \underset{g_{23}}{2}, g_{23} g_{31} g_{12}\right) \\
& \quad=W^{\prime}\left(g_{33}, g_{11}, g_{22}, \stackrel{g_{12}}{2}, \underset{g_{23}}{2}, \underset{g_{31}}{2}, g_{23} g_{31} g_{12}\right) .
\end{aligned}
$$

Taking

$$
\left(y_{1}, y_{2}, y_{3}, z_{1}, z_{2}, z_{3}\right)=\left(g_{11}, g_{22}, g_{33}, \underset{g_{23}}{2}, \stackrel{2}{g_{31}}, \stackrel{2}{g_{12}}\right)
$$

and

$$
\left(I_{1}, I_{2}, \cdots, I_{r}\right)=g_{23} g_{31} g_{12}
$$

in Theorems 3 and 4 , we see that $W^{\prime}$ and hence $W$ must be expressible as a polynomial in $K_{1}, K_{2}, \cdots, K_{14}$ and $g_{23} g_{31} g_{12}$, where

$$
\begin{aligned}
& K_{1}=g_{11}+g_{22}+g_{33}, \quad K_{2}=g_{22} g_{33}+g_{33} g_{11}+g_{11} g_{22} \text {, } \\
& K_{3}=g_{11} g_{22} g_{33}, \quad K_{4}=g_{23}^{2}+g_{31}^{2}+g_{12}^{2} \text {, }
\end{aligned}
$$

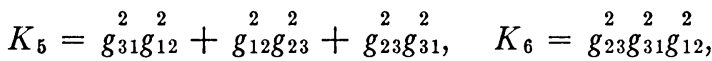

$$
\begin{aligned}
& K_{7}=g_{22} g_{12}^{2}+g_{33} g_{23}^{2}+g_{11} g_{31}^{2}, \quad K_{8}=g_{31}^{2} g_{33}+g_{12}^{2} g_{11}+g_{23}^{2} g_{22}, \\
& K_{9}=g_{33} g_{22}^{2}+g_{11} g_{33}^{2}+g_{22} g_{11}^{2}, \quad K_{10}=g_{12}^{2} g_{31}^{4}+g_{23}^{2} g_{12}^{4}+g_{31}^{2} g_{23}^{4}, \\
& K_{11}=g_{11} g_{31}^{2} g_{12}^{2}+g_{22} g_{12}^{2} g_{23}^{2}+g_{33} g_{23}^{2} g_{31}^{2}, \\
& K_{12}=g_{23}^{2} g_{22} g_{33}+g_{31}^{2} g_{33} g_{11}+g_{12}^{2} g_{11} g_{22}, \\
& K_{13}=g_{11} g_{22} g_{31}^{2}+g_{22} g_{33} g_{12}^{2}+g_{33} g_{11} g_{23}^{2},
\end{aligned}
$$

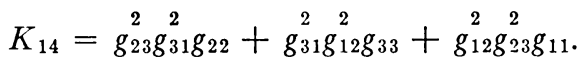

It is evident that $K_{6}$ is a redundant element. Hence a polynomial basis for these two classes is formed by the quantities $K_{1}$ to $K_{5}, K_{7}$ to $K_{14}$ and $g_{23} g_{31} g_{12}$.

(ii) Hextetrahedral, gyroidal and hexoctahedral classes.

For each of these classes the restrictions imposed on $W$ are obtained by substituting in (3.6) for $\bar{g}_{i j}$ from lines 2 to 24 respectively of Table 1 . The restrictions derived from lines 2,3 and 4 imply that $W$ must be expressible in the form

$$
W=W^{\prime}\left(g_{11}, g_{22}, g_{33}, \stackrel{2}{g_{23}}, \stackrel{2}{g_{31}}, \stackrel{2}{g_{12}}, g_{23} g_{31} g_{12}\right),
$$

where $W^{\prime}$ is a polynomial in the indicated variables. The remaining restrictions leave $g_{23} g_{31} g_{12}$ unaltered and imply that $W^{\prime}$ is unaltered by interchange of the pairs of variables $\left(g_{11}, g_{23}^{2}\right),\left(g_{22}, g_{31}^{2}\right)$ and $\left(g_{33}, g_{12}^{2}\right)$. Taking 


$$
\begin{aligned}
& \left(y_{1}, z_{1}\right)=\left(g_{11}, g_{23}^{2}\right), \quad\left(y_{2}, z_{2}\right)=\left(g_{22}, g_{31}^{2}\right), \\
& \left(y_{3}, z_{3}\right)=\left(g_{33}, g_{12}^{2}\right) \text { and }\left(I_{1}, I_{2}, \cdots, I_{r}\right)=g_{23} g_{31} g_{12}
\end{aligned}
$$

in Theorems 2 and 4 , we see that $W^{\prime}$ and hence $W$ must be expressible as a polynomial in $g_{23} g_{31} g_{12}$ and $J_{1}, J_{2}, \cdots, J_{9}$ where

$$
\begin{array}{ll}
J_{1}=g_{11}+g_{22}+g_{33}, & J_{2}=g_{22} g_{33}+g_{33} g_{11}+g_{11} g_{22}, \\
J_{3}=g_{11} g_{22} g_{33}, & J_{4}=g_{23}^{2}+g_{31}^{2}+g_{12}^{2}, \\
J_{5}=g_{31}^{2} g_{12}^{2}+g_{12}^{2} g_{23}^{2}+\underset{g_{23} g_{31}^{2},}{2} \quad J_{6}=\underset{g_{23} g_{31}^{2} g_{12}^{2},}{2} \\
J_{7}=g_{22}^{2} g_{22}^{2}+g_{33}^{2} g_{31}^{2}+g_{33}^{2} g_{23}^{2}+g_{11}^{2} g_{12}^{2}+g_{11}^{2} g_{31}^{2}+g_{22}^{2} g_{23}^{2}, \\
J_{8}=g_{11}^{2} g_{31}^{2} g_{12}^{2}+g_{22}^{2} g_{12}^{2} g_{23}^{2}+g_{33}^{2} g_{23}^{2} g_{31}^{2}, \\
J_{9}=g_{23}^{2} g_{22} g_{33}+g_{31}^{2} g_{33} g_{11}+g_{12}^{2} g_{11} g_{22} .
\end{array}
$$

Since $J_{6}$ is a redundant element, we see that the quantities $J_{1}$ to $J_{5}, J_{7}$ to $J_{9}$ and $g_{23} g_{31} g_{12}$ form a polynomial basis for the hextetrahedral, gyroidal and hexoctahedral classes.

We note that the elements of the polynomial bases of first and second degree in $g_{i j}$ are the same for all the classes of the cubic system. Consequently, if the displacement gradients, and hence the quantities $g_{i j}$, are sufficiently small so that terms of higher degree than the second in the expression for $W$ as a polynomial in $g_{i j}$ can be neglected, then $W$ takes the same form for all the classes of the cubic system.

10. The hexagonal system. For a material having hexagonal symmetry, the unit vectors $h_{i}$ are situated so that $h_{3}$ is perpendicular to the plane defined by $h_{1}$ and $h_{2}$, and so that $h_{1}$ can be made to coincide with $h_{2}$ by a rotation of $120^{\circ}$ about the direction of $h_{3}$. We take as our reference system a righthanded rectangular Cartesian coordinate system $x_{i}$, the $x_{1}$ and $x_{3}$ axes of which are parallel respectively to $h_{1}$ and $h_{3}$.

The hexagonal system contains twelve crystal classes which, together with the transformation groups characterizing them, are:

trigonal-pyramidal

rhombohedral

ditrigonal-pyramidal

trigonal-trapezohedral

hexagonal-scalenohedral

trigonal-dipyramidal

hexagonal-pyramidal

hexagonal-dipyramidal
$\left(I, S_{1}, S_{2}\right)$,

$\left(I, S_{1}, S_{2}, C, C S_{1}, C S_{2}\right)$,

$\left(I, S_{1}, S_{2}, R_{1}, R_{1} S_{1}, R_{1} S_{2}\right)$,

$\left(I, S_{1}, S_{2}, D_{1}, D_{1} S_{1}, D_{1} S_{2}\right)$,

$\left(I, S_{1}, S_{2}, C, C S_{1}, C S_{2}, R_{1}, R_{1} S_{1}, R_{1} S_{2}, D_{1}, D_{1} S_{1}\right.$, $\left.D_{1} S_{2}\right)$,

$\left(I, S_{1}, S_{2}, R_{3}, R_{3} S_{1}, R_{3} S_{2}\right)$,

$\left(I, S_{1}, S_{2}, D_{3}, D_{3} S_{1}, D_{3} S_{2}\right)$,

$\left(I, S_{1}, S_{2}, C, C S_{1}, C S_{2}, R_{3}, R_{3} S_{1}, R_{3} S_{2}, D_{3}, D_{3} S_{1}\right.$, $\left.D_{3} S_{2}\right)$, 
ditrigonal-dipyramidal (I, $S_{1}, S_{2}, R_{1}, R_{1} S_{1}, R_{1} S_{2}, R_{3}, R_{3} S_{1}, R_{3} S_{2}, D_{2}, D_{2} S_{1}$, $\left.D_{2} S_{2}\right)$,

dihexagonal-pyramidal $\quad\left(I, S_{1}, S_{2}, R_{1}, R_{1} S_{1}, R_{1} S_{2}, R_{2}, R_{2} S_{1}, R_{2} S_{2}, D_{3}, D_{3} S_{1}\right.$, $\left.D_{3} S_{2}\right)$,

hexagonal-trapezohedral ( $I, S_{1}, S_{2}, D_{1}, D_{1} S_{1}, D_{1} S_{2}, D_{2}, D_{2} S_{1}, D_{2} S_{2}, D_{3}, D_{3} S_{1}$, $\left.D_{3} S_{2}\right)$,

dihexagonal-dipyramidal ( $I, S_{1}, S_{2}, C, C S_{1}, C S_{2}, R_{1}, R_{1} S_{1}, R_{1} S_{2}, R_{2}, R_{2} S_{1}$, $R_{2} S_{2}, R_{3}, R_{3} S_{1}, R_{3} S_{2}, D_{1}, D_{1} S_{1}, D_{1} S_{2}, D_{2}, D_{2} S_{1}$, $\left.D_{2} S_{2}, D_{3}, D_{3} S_{1}, D_{3} S_{2}\right)$.

(i) Trigonal-pyramidal and rhombohedral classes.

For each of these classes, the restrictions on $W$ are obtained from (3.6) by assigning to $\bar{g}_{i j}$ the values given in lines 1 and 2 respectively of Table 2 . They may be written as

$$
\begin{aligned}
& W\left(g_{11}, g_{22}, g_{33}, g_{23}, g_{31}, g_{12}\right) \\
& =W\left[y_{2}, \frac{1}{3}\left(2 y_{1}-y_{2}+2 y_{3}\right), g_{33},-\frac{1}{3^{1 / 2}}\left(z_{1}-z_{3}\right), z_{2}, \frac{1}{3^{1 / 2}}\left(y_{1}-y_{3}\right)\right] \\
& =W\left[y_{3}, \frac{1}{3}\left(2 y_{1}+2 y_{2}-y_{3}\right), g_{33}, \frac{1}{3^{1 / 2}}\left(z_{1}-z_{2}\right), z_{3}, \frac{1}{3^{1 / 2}}\left(y_{2}-y_{1}\right)\right],
\end{aligned}
$$

where the notation of Equations (3.7) is used. From (3.7), we see that

$$
\begin{gathered}
g_{11}=y_{1}, g_{22}=\frac{1}{3}\left(2 y_{2}+2 y_{3}-y_{1}\right), \\
g_{12}=\frac{1}{3^{1 / 2}}\left(y_{3}-y_{2}\right), \\
g_{31}=z_{1} \text { and } g_{23}=\frac{1}{3^{1 / 2}}\left(z_{2}-z_{3}\right) .
\end{gathered}
$$

Introducing (10.2) into (10.1), and noting that we may regard $W$ as a polynomial $W^{\prime}$ (say) in $y_{1}, y_{2}, y_{3}, z_{1}, z_{2}, z_{3}$ and $g_{33}$, we see that (10.1) may be rewritten as

$$
\begin{aligned}
& W^{\prime}\left(y_{1}, y_{2}, y_{3}, z_{1}, z_{2}, z_{3}, g_{33}\right) \\
& \quad=W^{\prime}\left(y_{2}, y_{3}, y_{1}, z_{2}, z_{3}, z_{1}, g_{33}\right) \\
& \quad=W^{\prime}\left(y_{3}, y_{1}, y_{2}, z_{3}, z_{1}, z_{2}, g_{33}\right) .
\end{aligned}
$$

Employing Theorems 3 and 4, we see that a polynomial basis for $W^{\prime}$ and hence for $W$ is formed by $g_{33}$ and $K_{1}, K_{2}, \cdots, K_{14}$, where $K_{1}, K_{2}, \cdots, K_{14}$ are obtained from Equations (4.3) by substituting for $y_{1}, y_{2}, y_{3}, z_{1}, z_{2}, z_{3}$ from (3.7). It can be shown that if $W^{\prime}$ is expressible as a polynomial in $g_{33}, K_{1}$, $K_{2}, \cdots, K_{14}$ it is also expressible as a polynomial in 


$$
\begin{aligned}
& g_{33}, g_{11}+g_{22}, g_{11} g_{22}-g_{12}^{2}, g_{11}\left[\left(g_{11}+3 g_{22}\right)^{2}-12 g_{12}^{2}\right], \\
& g_{31}^{2}+g_{23}^{2}, g_{31}\left(g_{31}^{2}-3 g_{23}^{2}\right),\left(g_{11}-g_{22}\right) g_{31}-2 g_{12} g_{23}, \\
& \left(g_{22}-g_{11}\right) g_{23}-2 g_{12} g_{31}, 3 g_{12}\left(g_{11}-g_{22}\right)^{2}-4 g_{12}^{3}, \\
& g_{23}\left(g_{23}^{2}-3 g_{31}^{2}\right), g_{22} g_{31}^{2}+g_{11} g_{23}^{2}-2 g_{23} g_{31} g_{12}, \\
& g_{31}\left[\left(g_{11}+g_{22}\right)^{2}+4\left(g_{12}^{2}-g_{22}^{2}\right)\right]-8 g_{11} g_{12} g_{23} . \\
& g_{23}\left[\left(g_{11}+g_{22}\right)^{2}+4\left(g_{12}^{2}-g_{22}^{2}\right)\right]+8 g_{11} g_{12} g_{31}, \\
& \left(g_{11}-g_{22}\right) g_{23} g_{31}+g_{12}\left(g_{23}^{2}-g_{31}^{2}\right)
\end{aligned}
$$

and that any polynomial in the quantities (10.4) may be expressed as a polynomial in $g_{33}$ and $K_{1}, K_{2}, \cdots, K_{14}$. These results can be readily obtained by inspection if we express each of the quantities $g_{33}, K_{1}, K_{2}, \cdots, K_{14}$ as a polynomial in the quantities (10.4). Thus, the expressions (10.4) form a polynomial basis for the trigonal-pyramidal and rhombohedral classes.

(ii) Ditrigonal-pyramidal, trigonal-trapezohedral and hexagonal-scalenohedral classes.

For each of these classes, the restrictions imposed on $W$ are obtained from (3.6) by assigning to $\bar{g}_{i j}$ the values given in line 2 of Table 1 and lines $1,2,3$ and 4 respectively of Table 2 . As in the case of the trigonal-pyramidal and rhombohedral classes, we regard $W$ as a polynomial $W^{\prime}$ in $y_{1}, y_{2}, y_{3}, z_{1}, z_{2}, z_{3}$ and $g_{33}$ defined by (3.7) and obtain the limitations imposed on $W^{\prime}$. We then employ the notation

$$
Y_{1}=y_{1}, \quad Y_{2}=y_{2}, \quad Y_{3}=y_{3}, \quad Z_{1}=\frac{1}{3^{1 / 2}}\left(z_{2}-z_{3}\right),
$$

$$
Z_{2}=\frac{1}{3^{1 / 2}}\left(z_{3}-z_{1}\right), \quad Z_{3}=\frac{1}{3^{1 / 2}}\left(z_{1}-z_{2}\right)
$$

and consider $W^{\prime}$ and hence $W$ as a polynomial $W^{\prime \prime}$ (say) in $Y_{1}, Y_{2}, Y_{3}, Z_{1}, Z_{2}$, $Z_{3}$ and $g_{33}$. The restrictions on the form of $W^{\prime \prime}$ then take the form that it is unaltered by interchange of any pair of the variables $\left(Y_{1}, Z_{1}\right),\left(Y_{2}, Z_{2}\right)$, $\left(Y_{3}, Z_{3}\right)$ while the dependence on $g_{33}$ involves no restrictions; i.e.,

$$
\begin{aligned}
W^{\prime \prime} & \left(Y_{1}, Y_{2}, Y_{3}, Z_{1}, Z_{2}, Z_{3}, g_{33}\right) \\
& =W^{\prime \prime}\left(Y_{1}, Y_{3}, Y_{2}, Z_{1}, Z_{3}, Z_{2}, g_{33}\right) \\
& =\text { etc. }
\end{aligned}
$$

Then we see from Theorems 2 and 4 that the quantities $g_{33}, J_{1}, J_{2}, \cdots, J_{9}$ form a polynomial basis for $W^{\prime \prime}$, and hence for $W$, if we replace $y_{1}, y_{2}, \cdots, z_{3}$ by $Y_{1}, Y_{2}, \cdots, Z_{3}$ in the expressions (4.2) for $J_{1}, J_{2}, \cdots, J_{9}$. By expressing 
each of the quantities $g_{33}, J_{1}, J_{2}, \cdots, J_{9}$ so obtained as a polynomial in $g_{i j}$ ind then as a polynomial in the quantities

$$
\begin{aligned}
& g_{33}, g_{11}+g_{22}, g_{11} g_{22}-g_{12}^{2}, g_{11}\left[\left(g_{11}+3 g_{22}\right)^{2}-12 g_{12}^{2}\right] \\
& g_{31}^{2}+g_{23}^{2}, g_{23}\left(g_{23}^{2}-3 g_{31}^{2}\right),\left(g_{11}-g_{22}\right) g_{23}+2 g_{12} g_{31}, \\
& g_{11} g_{31}^{2}+g_{22} g_{23}^{2}+2 g_{23} g_{31} g_{12}, \\
& g_{23}\left[\left(g_{11}+g_{22}\right)^{2}-4\left(g_{22}^{2}-g_{12}^{2}\right)\right]+8 g_{11} g_{12} g_{31},
\end{aligned}
$$

it can be readily seen that any polynomial in $g_{33}, J_{1}, J_{2}, \cdots, J_{9}$ can be expressed as a polynomial in the quantities (10.7) and conversely any polynomial in the quantities (10.7) can be expressed as a polynomial in $g_{33}$, $J_{1}, J_{2}, \cdots, J_{9}$. Thus, the expressions (10.7) form a polynomial basis for the ditrigonal-pyramidal, trigonal-trapezohedral and hexagonal-scalenohedral classes.

(iii) Trigonal-dipyramidal, hexagonal-pyramidal and hexagonal-dipyramidal classes.

For each of these classes, the restrictions imposed on $W$ are obtained from (3.6) by assigning to $\bar{g}_{i j}$ the values given in line 4 of Table 1 and lines $1,2,7$ and 8 respectively of Table 2 . It can be shown in a manner analogous to that adopted in discussing the monoclinic system, that if $W$ satisfies the restriction derived from line 4 of Table 1 , it must be expressible in the form

$$
W=W^{\prime}\left(g_{11}, g_{22}, g_{33}, \stackrel{2}{g_{23}}, \stackrel{2}{g_{31}}, g_{12}, g_{23} g_{31}\right)
$$

where $W^{\prime}$ is a polynomial. We now employ the notation

$$
\text { (10.9) } Y_{1}=y_{1}, Y_{2}=y_{2}, Y_{3}=y_{3}, Z_{1}=z_{1}^{2}, Z_{2}=z_{2}^{2} \text { and } Z_{3}=z_{3}^{2}
$$

in (10.8), where $y_{1}, y_{2}, \cdots, z_{3}$ are defined by (3.7). It is then seen that $W^{\prime}$ may be considered as a polynomial $W^{\prime \prime}$ (say) in $Y_{1}, Y_{2}, \cdots, Z_{3}$ and $g_{33}$. Introducing into $W^{\prime \prime}$ the restrictions derived from lines $1,2,7$ and 8 of Table 2, we obtain

$$
\begin{aligned}
& W^{\prime \prime}\left(Y_{1}, Y_{2}, Y_{3}, Z_{1}, Z_{2}, Z_{3}, g_{33}\right) \\
& \quad=W^{\prime \prime}\left(Y_{2}, Y_{3}, Y_{1}, Z_{2}, Z_{3}, Z_{1}, g_{33}\right) \\
& \quad=W^{\prime \prime}\left(Y_{3}, Y_{1}, Y_{2}, Z_{3}, Z_{1}, Z_{2}, g_{33}\right) .
\end{aligned}
$$

Employing Theorems 3 and 4 , we see that $g_{33}$ and the quantities $K_{1}, K_{2}, \cdots$, $K_{14}$ defined by (4.3), with $Y_{1}, Y_{2}, \cdots, Z_{3}$ substituted for $y_{1}, y_{2}, \cdots, z_{2}$, form a polynomial basis for $W^{\prime \prime}$ and hence for $W$. Each of the quantities $g_{33}, K_{1}, K_{2}, \cdots, K_{14}$ may be expressed as a polynomial in the quantities $g_{i j}$ by substitution from (10.9) and (3.7). Then, by expressing each of the quan- 
tities $g_{33}, K_{1}, K_{2}, \cdots, K_{14}$ as a polynomial in the quantities

$(10.11)$

$$
\begin{aligned}
& g_{33}, g_{11}+g_{22}, g_{11} g_{22}-g_{12}^{2}, g_{11}\left[\left(g_{11}+3 g_{22}\right)^{2}-12 g_{12}^{2}\right], \\
& g_{31}^{2}+g_{23}^{2}, g_{31}^{2}\left(g_{31}^{2}-3 g_{23}^{2}\right)^{2}, g_{11} g_{23}^{2}+g_{22}^{2} g_{31}^{2}-2 g_{23} g_{31} g_{12}, \\
& g_{12}\left(g_{31}^{2}-g_{23}^{2}\right)+\left(g_{22}-g_{11}\right) g_{31} g_{23}, 3 g_{12}\left(g_{11}-g_{22}\right)^{2}-4 g_{12}^{3}, \\
& g_{31} g_{23}\left[3\left(g_{31}^{2}-g_{23}^{2}\right)^{2}-4 g_{31}^{2} g_{23}^{2}\right], \\
& g_{11}\left(g_{31}^{4}+3 g_{23}^{4}\right)+2 g_{22}^{2} g_{31}^{2}\left(g_{31}^{2}+3 g_{23}^{2}\right)-8 g_{12} g_{23} g_{31}^{3}, \\
& g_{31}^{2}\left[\left(g_{11}+g_{22}\right)^{2}-4\left(g_{22}^{2}-g_{12}^{2}\right)\right]-2 g_{11}\left[\left(g_{11}+3 g_{22}\right)\left(g_{31}^{2}+g_{23}^{2}\right)-4 g_{23} g_{31} g_{12}\right], \\
& g_{23} g_{31}\left[\left(g_{11}+g_{22}\right)^{2}-4\left(g_{22}^{2}-g_{12}^{2}\right)\right]+4 g_{11} g_{12}\left(g_{23}^{2}-g_{31}^{2}\right), \\
& g_{12}\left[\left(g_{31}^{2}+g_{23}^{2}\right)^{2}+4 g_{23}^{2}\left(g_{31}^{2}-g_{23}^{2}\right)\right]-4 g_{31}^{3} g_{23}\left(g_{11}-g_{22}\right),
\end{aligned}
$$

we see that the quantities (10.11) form a polynomial basis for $W$ for the trigonal-dipyramidal, hexagonal-pyramidal and hexagonal-dipyramidal classes.

(iv) Ditrigonal-dipyramidal, dihexagonal-pyramidal, hexagonal-trapezohedral and dihexagonal-dipyramidal classes.

For each of these classes the restrictions imposed on $W$ are obtained from (3.6) by assigning to $\bar{g}_{i j}$ the values given in lines 2,3 and 4 of Table 1 and lines $1,2, \cdots, 8$ of Table 2 respectively. It can be shown in the manner adopted in discussing the preceding three classes of the hexagonal system that if $W$ satisfies the restrictions derived from line 4 of Table 1 and lines $1,2,7$ and 8 of Table 2, it must be expressible as a polynomial $W^{\prime \prime}$ in $g_{33}$ and the quantities $Y_{1}, Y_{2}, Y_{3}, Z_{1}, Z_{2}, Z_{3}$, defined by (10.9) and (3.7), which satisfies the relations (10.10). In a similar manner it can be shown that the restrictions on $W$ derived from lines 2 and 3 of Table 1 and lines 3, 4, 5 and 6 of Table 2 impose three further restrictions on $W^{\prime \prime}$. These are

$$
\begin{aligned}
& W^{\prime \prime}\left(Y_{1}, Y_{2}, Y_{3}, Z_{1}, Z_{2}, Z_{3}, g_{33}\right) \\
& \quad=W^{\prime \prime}\left(Y_{1}, Y_{3}, Y_{2}, Z_{1}, Z_{3}, Z_{2}, g_{33}\right) \\
& \quad=W^{\prime \prime}\left(Y_{2}, Y_{1}, Y_{3}, Z_{2}, Z_{1}, Z_{3}, g_{33}\right) \\
& \quad=W^{\prime \prime}\left(Y_{3}, Y_{2}, Y_{1}, Z_{3}, Z_{2}, Z_{1}, g_{33}\right) .
\end{aligned}
$$

The relations (10.10) and (10.12) together imply that $W^{\prime \prime}$ must be symmetric in the three pairs of variables $\left(Y_{1}, Z_{1}\right),\left(Y_{2}, Z_{2}\right)$ and $\left(Y_{3}, Z_{3}\right)$. We see from Theorems 2 and 4 that $g_{33}$ and $J_{1}, J_{2}, \cdots, J_{9}$, defined by (4.2) with $y_{1}, y_{2}, \cdots, z_{3}$ replaced by $Y_{1}, Y_{2}, \cdots, Z_{3}$, form a polynomial basis for $W^{\prime \prime}$ and hence for $W$. Expressing $g_{33}, J_{1}, J_{2}, \cdots, J_{9}$ as polynomials in $g_{i j}$ and then in the quantities 


$$
\begin{aligned}
& g_{33}, g_{11}+g_{22}, g_{11} g_{22}-g_{12}^{2}, g_{11}\left[\left(g_{11}+3 g_{22}\right)^{2}-12 g_{12}^{2}\right], \\
& g_{31}^{2}+g_{23}^{2}, g_{31}^{2}\left(g_{31}^{2}-3 g_{23}^{2}\right)^{2}, g_{11} g_{23}^{2}+g_{22} g_{31}^{2}-2 g_{23} g_{31} g_{12}, \\
& g_{11}\left(g_{31}^{4}+3 g_{23}^{4}\right)+2 g_{22} g_{31}^{2}\left(g_{31}^{2}+3 g_{23}^{2}\right)-8 g_{12} g_{23} g_{31}^{3}, \\
& g_{31}^{2}\left[\left(g_{11}+g_{22}\right)^{2}-4\left(g_{22}^{2}-g_{12}^{2}\right)\right]-2 g_{11}\left[\left(g_{11}+3 g_{22}\right)\left(g_{31}^{2}+g_{23}^{2}\right)-4 g_{23} g_{31} g_{12}\right]
\end{aligned}
$$

it is readily seen analogously with the previous cases, that the quantities (10.13) form a polynomial basis for the ditrigonal-dipyramidal, dihexagonalpyramidal, hexagonal-trapezohedral and dihexagonal-dipyramidal classes.

We note that the elements of first and second degree in $g_{i j}$ of the polynomial bases for the crystal classes discussed in (iii) and (iv) are the same. Consequently, if the displacement gradients, and hence the quantities $g_{i j}$, are sufficiently small so that terms of higher degree than the second in the expression for $W$ as a polynomial in $g_{i j}$ can be neglected, then $W$ takes the same form for all the crystal classes discussed in (iii) and (iv).

11. Appendix. In the introduction it is assumed that if a body of the material considered is deformed so that a particle initially at $X_{i}^{\prime}$ in the rectangular Cartesian coordinate system $x_{i}^{\prime}$ moves to $x_{i}^{\prime}$ in the same coordinate system, then the strain-energy function $W$, defined as the strain-energy per unit volume measured in the undeformed state, may be expressed as a polynomial in the quantities $g_{i j}^{\prime}$ defined by equation (1.1). It is the purpose of this section to discuss this assumption further.

If we assume initially that $W$ is expressible as a polynomial in the gradients $\partial x_{i}^{\prime} / \partial X_{j}^{\prime}$, then $W$ is unaltered if the deformed body is subjected to an arbitrary rigid-body rotation in which the particle at $x_{i}^{\prime}$ moves to $x_{i}^{\prime \prime}$, where

$$
x_{i}^{\prime \prime}=a_{i k} x_{k}^{\prime}
$$

with

$$
a_{i j} a_{i k}=\delta_{j k} \quad \text { and } \quad \operatorname{det} a_{i j}=1
$$

We therefore have

$$
W\left(\partial x_{i}^{\prime} / \partial X_{j}^{\prime}\right)=W\left(\partial x_{i}^{\prime \prime} / \partial X_{j}^{\prime}\right)=W\left(a_{i k} \partial x_{k}^{\prime} / \partial X_{j}^{\prime}\right),
$$

where $W$ is a polynomial function of the indicated variables, for all $a_{i j}$ satisfying the relations (11.2). It follows from a well-known theorem in the theory of invariants (see, for example, H. Weyl, 1946, p. 53 et seq.) that $W$ must be expressible as a polynomial in the quantities $g_{i j}^{\prime}+\delta_{i j}$ defined by (1.1) and $\operatorname{det} \partial x_{i}^{\prime} / \partial X_{j}^{\prime}$. Now, it is easily seen that

$$
\operatorname{det}\left(g_{i j}^{\prime}+\delta_{i j}\right)=\left(\operatorname{det} \partial x_{i}^{\prime} / \partial X_{j}^{\prime}\right)^{2}
$$


and, since for any deformation which is possible in a real material det $\partial x_{i}^{\prime} / \partial X_{j}^{\prime}$ is positive, we have

$$
\operatorname{det} \partial x_{i}^{\prime} / \partial X_{j}^{\prime}=\left[\operatorname{det}\left(g_{i j}^{\prime}+\delta_{i j}\right)\right]^{1 / 2} .
$$

Also, since for any deformation which is possible in a real material $\operatorname{det}\left(g_{i j}^{\prime}+\delta_{i j}\right)$ cannot be zero, $\left[\operatorname{det}\left(g_{i j}^{\prime}+\delta_{i j}\right)\right]^{1 / 2}$ may be approximated to any desired accuracy by a polynomial in $\operatorname{det}\left(g_{i j}^{\prime}+\delta_{i j}\right)$. Thus, $W$ may be approximated to any desired accuracy by a polynomial in the quantities $g_{i j}^{\prime}+\delta_{i j}$ and hence in the quantities $g_{i j}^{\prime}$.

We have already noted that it follows from our initial assumption that $W$ must be expressible precisely as a polynomial in the quantities $g_{i j}^{\prime}+\delta_{i j}$ and det $\partial x_{i}^{\prime} / \partial X_{j}^{\prime}$. Hence, it must be expressible precisely as a polynomial in the quantities $g_{i j}^{\prime}$ and $\operatorname{det} \partial x_{i}^{\prime} / \partial X_{j}^{\prime}$. If such an expression is made the starting point for the discussion of the various crystal classes, then we have only to add to the basic invariants in terms of which $W$ is expressed in each case the expression $\operatorname{det} \partial x_{i}^{\prime} / \partial X_{j}^{\prime}$, since the latter is invariant under each of the transformations considered.

\section{REFERENCES}

1. F. Birch, Physical Review vol. 71 (1947) pp. 809-824.

2. J. D. Dana and C. S. Hurlbut, Dana's textbook of mineralogy, New York, John Wiley and Sons, 1952.

3. A. E. Green and E. W. Wilkes, Journal of Rational Mechanics and Analysis vol. 3 (1954) pp. 713-723.

4. A. E. Green and W. Zerna, Theoretical elasticity, Oxford, Clarendon Press, 1954.

5. F. D. Murnaghan, Finite deformation of an elastic solid, New York, John Wiley \& Sons, 1951.

6. P. L. Sheng, Secondary elcasticity, Chinese Association for the Advancement of Science Monographs, no. 1, 1955.

7. C. Truesdell, Journal of Rational Mechanics and Analysis vol. 1 (1952) pp. 125-300.

8. W. Voigt, Lehrbuch der Kristallphysik, Leipzig, B. G. Teubner, 1910.

9. H. Weyl, The classical groups, their invariants and representations, Princeton, Princeton University Press, 1946.

BROWN UNIVERSITY, Providence, R. I. 